\title{
Label-free quantitative cell division monitoring of endothelial cells by digital holographic microscopy
}

\author{
Björn Kemper*, \\ University of Münster \\ Center for Biomedical Optics and Photonics \\ Robert-Koch-Strasse 45 \\ Münster, 48149 Germany
}

\section{Andreas Bauwens* \\ University of Münster \\ Institute of Hygiene \\ Robert-Koch-Strasse 41 \\ Münster, 48149 Germany}

\section{Angelika Vollmer \\ Steffi Ketelhut}

Patrik Langehanenberg

University of Münster

Center for Biomedical Optics and Photonics

Robert-Koch-Strasse 45

Münster, 48149 Germany

\section{Johannes Müthing \\ Helge Karch}

University of Münster

Institute of Hygiene

Robert-Koch-Strasse 41

Münster, 48149 Germany

\section{Gert von Bally}

University of Münster

Center for Biomedical Optics and Photonics

Robert-Koch-Strasse 45

Münster, 48149 Germany

\begin{abstract}
Digital holographic microscopy (DHM) enables quantitative multifocus phase contrast imaging for nondestructive technical inspection and live cell analysis. Time-lapse investigations on human brain microvascular endothelial cells demonstrate the use of DHM for label-free dynamic quantitative monitoring of cell division of mother cells into daughter cells. Cytokinetic DHM analysis provides future applications in toxicology and cancer research. () 2010 Society of PhotoOptical Instrumentation Engineers. [DOI: 10.1117/1.3431712]
\end{abstract}

Keywords: quantitative phase contrast imaging; digital holographic microscopy; live cell imaging; mitosis; cell cycle; cell tracking.

Paper 09458R received Oct. 14, 2009; revised manuscript received Mar. 25, 2010; accepted for publication Apr. 1, 2010; published online Jun. 1, 2010.

\section{Introduction}

Digital holographic microscopy (DHM) enables label-free, quantitative phase contrast imaging for high-resolution technical inspection and minimally invasive live cell analysis ${ }^{1-7}$ that is also suitable for modular add-on integration into commercial microscopes. ${ }^{8}$ Compared to other phase contrast methods, ${ }^{9,10}$ interferometry-based techniques, ${ }^{10-15}$ and optical coherence tomography or optical coherence microscopy, ${ }^{16-21}$ DHM provides quantitative phase contrast with subsequent numerical focus correction (multifocus imaging) from a single recorded hologram. In combination with algorithms for the quantification of the image sharpness, numerical autofocusing without mechanical focus realignment is possible. ${ }^{22}$ These DHM features are of particular advantage for measurements on cellular specimens with high-magnification optics and for

*Contributed equally as first authors.

${ }^{+}$Address all correspondence to Björn Kemper, University of Münster, Center for Biomedical Optics and Photonics, Robert-Koch-Strasse 45, Münster, 48149 Germany. Tel: 49-25-1835-6888. Fax: 49-25-183-58536; E-mail: bkemper@unimuenster.de the detection of fast processes. Furthermore, long-term measurements, where focus tracking is required due to mechanical instability or thermal effects, ${ }^{23}$ and 3-D tracking of living cells $^{24}$ are enabled. Here, we demonstrate the use of DHM for label-free quantitative dynamic monitoring of endothelial cell division.

\section{Materials and Methods}

In order to explore the applicability of DHM for the quantitative analysis of cell division, investigations on human brain microvascular endothelial cells (HBMECs) ${ }^{25}$ were performed. For the experiments, the HBMECs were cultivated in a Petri dish (ibidi $\mu$-Dish, ibidi GmbH, Munich, Germany) using Leibowitz L-15 medium (PAA, Pasching, Austria) supplemented with $10 \% \mathrm{Nu}$-Serum (Becton Dickinson Biosciences, Bedford, Massachusetts), 10\% fetal calf serum (FCS-Gold, PAA, Pasching, Austria), $1 \mathrm{mM}$ sodium pyruvate, $2 \mathrm{mM}$ L-glutamine, $1 \%$ nonessential amino acids $(100 \times)$, and $1 \%$

1083-3668/2010/15(3)/036009/6/\$25.00 @ 2010 SPIE 


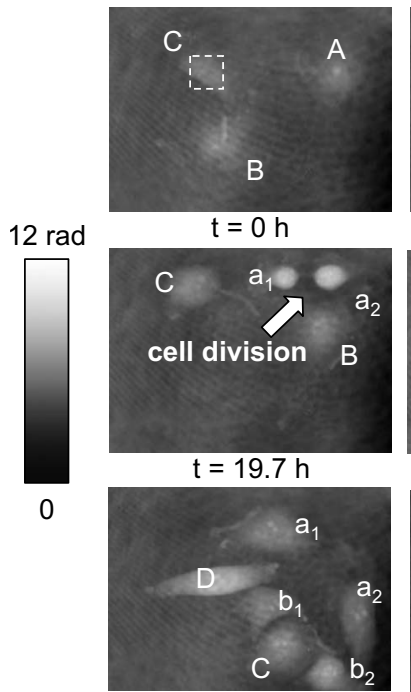

$t=36.8 h$

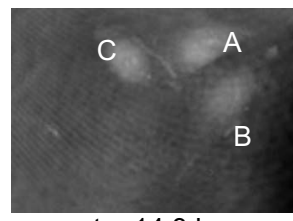

$\mathrm{t}=14.3 \mathrm{~h}$

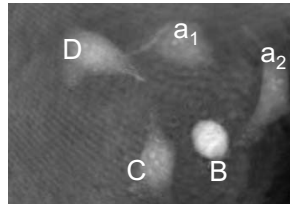

$\mathrm{t}=32.4 \mathrm{~h}$

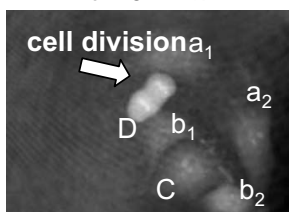

$t=37.7 h$

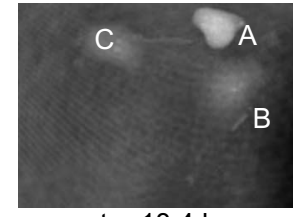

$t=18.4 h$

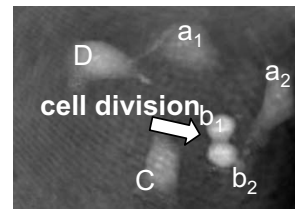

$t=32.5 h$

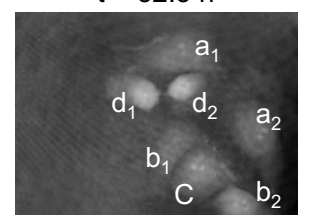

$\mathrm{t}=38.4 \mathrm{~h}$

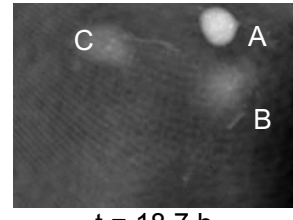

$\mathrm{t}=18.7 \mathrm{~h}$

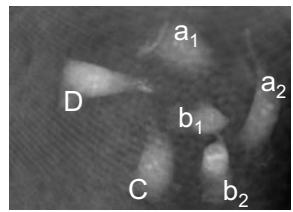

$\mathrm{t}=33.5 \mathrm{~h}$

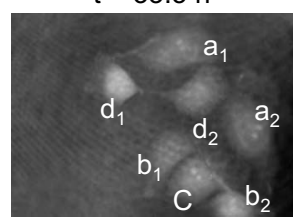

$\mathrm{t}=38.8 \mathrm{~h}$

Fig. 1 Time-dependent quantitative DHM phase contrast images of living HBMECs (coded to 256 gray levels). The arrows indicate cell division after $t=19.7 \mathrm{~h}$ (cell A), $t=35.5 \mathrm{~h}$ (cell B), and $t=37.7 \mathrm{~h}$ (cell D). For cell C, no cell division is observed during the experimental period. The corresponding daughter cells after the cell division process are denoted as $a_{1}, a_{2}, b_{1}, b_{2}, d_{1}$, and $d_{2}$. The typical size of the ROI that was used for data evaluation for two-dimensional cell tracking is indicated in the phase contrast image for $t=0$ by a dashed box around cell $\mathrm{C}$.

vitamin mix $(100 \times)$ (all from Lonza, Cologne, Germany). The cells were observed with an inverse microscope (iMIC, Till Photonics, Gräfelfing, Germany) with attached DHM module that is based on a principle described in Ref. 8. The coherent light source was a frequency-doubled Nd:YAG laser (Compass 315M-100, Coherent, Lübeck, Germany, $\lambda$ $=532 \mathrm{~nm}$ ). A climate chamber (Solent Scientific Ltd., Segensworth, UK) was utilized for temperature stabilization at $37^{\circ} \mathrm{C}$. In a time-lapse series, digital off-axis holograms of selected cells were recorded every $3 \mathrm{~min}$ over a period of $45 \mathrm{~h}$ with a charge-coupled device sensor (CCD, 1280 $\times 960$ pixels, The Imaging Source DMK 41BF02, Bremen, Germany). For imaging, a $40 \times$ microscope lens (Zeiss A-Plan $40 \times / 0.65)$ was used. The numerical evaluation of the resulting 900 digital holograms was performed by spatial phase shifting reconstruction, as described in Refs. 2 and 26. From the resulting quantitative phase contrast images, the lateral position of the cells and the maximum cell thickness were determined. ${ }^{24}$ Therefore, in a first step, the phase distributions were low-pass-filtered two times, with a box average filter of $5 \times 5$ pixels. In this way, substructures of the specimen in the phase distributions and noise-e.g., due to parasitic interferences and coherent noise-were reduced. Afterward, within a region of interest (ROI) in which the analyzed cell was located, the pixel coordinates of the maximum phase contrast were determined. The automated tracking of dynamic displacements of time-lapse sequences was performed by successive recentering of the ROI to the coordinates of the preceding maximal phase value. ${ }^{24}$ By calibration of the imaging scale with a USAF 1951 test chart, the resulting lateral displacement trajectories of the cells in pixel coordinates were converted to metric units. During the cell tracking procedure, in addition to the lateral $x, y$-coordinates of the cell position, the maximum phase contrast $\Delta \varphi_{\text {cell,max }}(x, y)$ was obtained. From $\Delta \varphi_{\text {cell,max }}(x, y)$, the maximum cell thickness $d_{\text {cell,max }}(x, y)$ was calculated $^{3,26}$.

$$
d_{\text {cell,max }}(x, y)=\frac{\lambda \Delta \varphi_{\text {cell, } \max }(x, y)}{2 \pi} \cdot \frac{1}{n_{\text {cell }}-n_{\text {medium }}} .
$$

The integral cellular refractive index $n_{\text {cell }}$ in Eq. (1) was estimated by the average value $n_{\text {cell }}=1.373 \pm 0.005$ that was retrieved from DHM measurements on HBMECs in suspension $(N=54$; see detailed description of the procedure in Ref. 27). The refractive index of the cell culture medium was determined by an Abbe refractometer (WYA-2W, Hinotek Ltd, Ningbo, China) to $n_{\text {medium }}=1.337 \pm 0.001$.

\section{Results}

Figure 1 shows representative results for the obtained quantitative DHM phase contrast images (coded to 256 gray levels). Video 1 presents a fast-motion movie of the phase contrast images for the whole experimental period. After $t=19.7 \mathrm{~h}$, $t=32.5 \mathrm{~h}$, and $t=37.7 \mathrm{~h}$, the HBMECs denoted as A, B, and $\mathrm{D}$, respectively, underwent a cell division. The corresponding

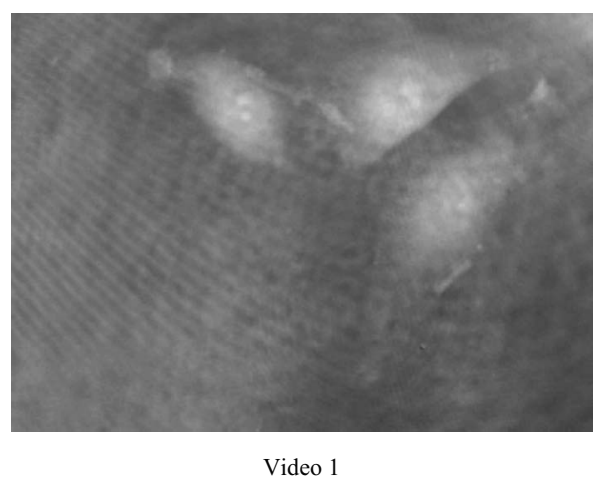

Video 1 Fast-motion movie of quantitative DHM phase contrast images of living HBMECs for the whole experimental period of $45 \mathrm{~h}$ (2.1 MB, QuickTime). [URL: http://dx.doi.org/10.1117/1.3431712.1]. 


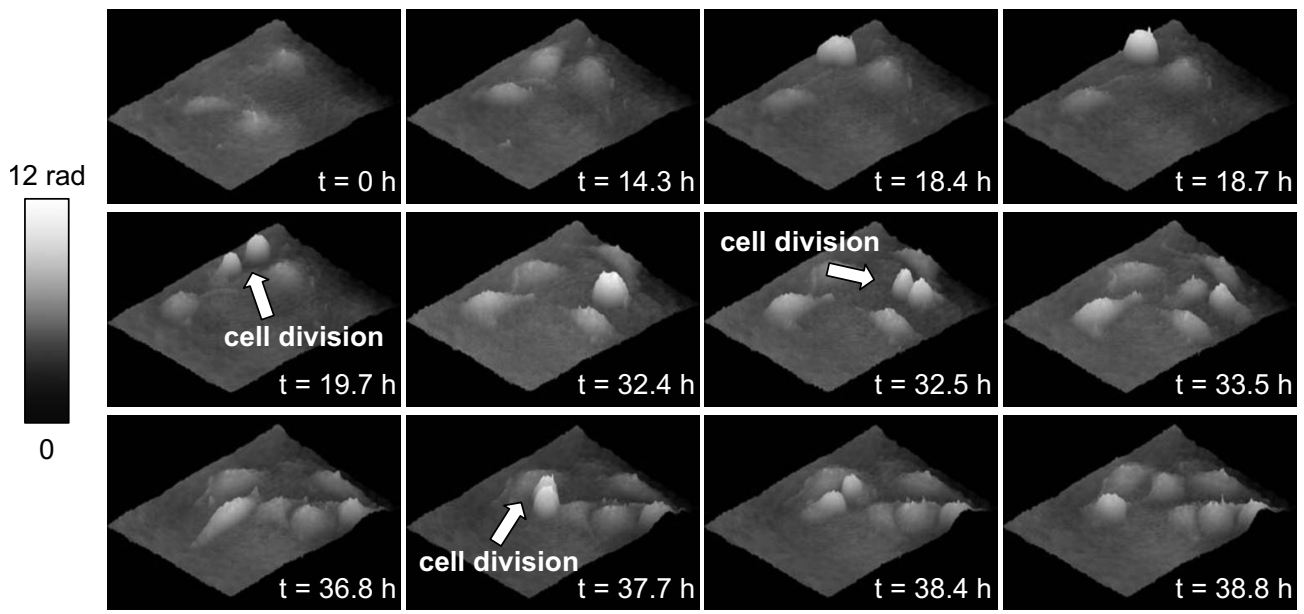

Fig. 2 Gray-level-coded pseudo-3-D representation of the quantitative contrast images of living HBMECs in Fig. 1.

daughter cells after the cell division process are designated as $\mathrm{a}_{1}, \mathrm{a}_{2}, \mathrm{~b}_{1}, \mathrm{~b}_{2}$, and $\mathrm{d}_{1}, \mathrm{~d}_{2}$. Cell $\mathrm{C}$ did not divide during the experimental period. It was observed that the phase contrast increases significantly before the cell division process. During the cell cycle phases in which the cells adhere on the substrate subcellular regions with a higher density than that of the surrounding areas like the nucleus, the nuclear membrane and the nucleoli become visible. In analogy, during mitosis, bright areas in the phase contrast images indicate the separation of the chromosomes - in particular, for cell $\mathrm{D}$ at $t=37.7 \mathrm{~h}$. Figure 2 shows a gray-level coded pseudo-3-D representation of the quantitative contrast images in Fig. 1. For cell cycle phases in which the cells adhere on the substrate, the quantitative phase contrast images correspond to the cell shape, ${ }^{26}$ while during cell rounding, the projection of the cell thickness is measured. ${ }^{28}$

For further evaluation of the DHM phase contrast images, the maximum phase contrast $\Delta \varphi_{\text {cell,max }}$, the maximum cell thickness $d_{\text {cell,max }}$, and the cell migration trajectories of all cells were determined, as described in Sec. 2 . The typical size of the ROI that was used for two-dimensional cell tracking is indicated in Fig. 1 in the DHM phase contrast image for $t$ $=0$ by a dashed box around cell $C$. During the evaluation procedure, the trajectories of the remaining daughter cells that were not detected by the automated cell tracking algorithm were started by manual selection after the cell division process.

Figure 3 shows for each cell the temporal dependence of the maximum phase contrast $\Delta \varphi_{\text {cell,max }}$ as well as the corresponding cell thickness $d_{\text {cell,max }}$ that is obtained from Eq. (1). Prior to the cell division, for cells A, B, and D, cell rounding is induced, which causes a significant increase of the maximum phase contrast and the cell thickness. The resulting peaks in the plots in Fig. 3 are marked with arrows. After the cell division for all cells, a decrease of $\Delta \varphi_{\text {cell,max }}$ and $d_{\text {cell,max }}$ is detected. For cell $\mathrm{C}$, which undergoes no cell division, only a few fluctuations of phase contrast and cell thickness are observed. Figure 4 shows the automated obtained cell migration $x$ - $y$-trajectories for all evaluated cells and demonstrates the reliability of the applied cell tracking algorithm.

\section{Discussion and Conclusions}

The results demonstrate the capabilities of DHM for dynamic cell division monitoring by simultaneous cell thickness measurement and two-dimensional cell tracking. The cell division is clearly detected by the phase contrast images in Figs 1 and 2 , and by the temporal dependency of phase contrast and cell thickness demonstrated in Fig. 3. However, as slight changes of the integral cellular refractive index cannot be completely excluded during the cell division process, the obtained values for the cell thickness in Fig. 3 have to be handled carefully. ${ }^{26}$ A further increase of the measurement accuracy for the cell thickness may be achieved by DHM-based decoupling procedures $^{29,30}$ but requires more complex experimental equipment or measurements with different light wave-lengths and a suitable dispersive dye. As the $x, y$-position data in Fig. 4 are obtained by the determination of the coordinates of the maximum phase contrast, the precision of the applied algorithm can be estimated to be sensitive to cell thickness fluctuations. Furthermore, the magnification of the applied experimental setup and disturbances in the phase contrast images due to scattering effects, e.g., by the cell culture medium, have to be taken into account. Thus, the error for the detection of the lateral cell position was estimated by the fluctuations of the curves in Fig. 4, which are found in the range of $1 \mu \mathrm{m}$ to $2 \mu \mathrm{m}$. These values are specific for the applied measurements setup and amount in the range of about $10 \%$ of the average lateral cell diameter. This is low in view of the cellshape changes that are observed in the DHM phase contrast images in Fig. 1 and can be explained as follows: For the cell cycle phases in which the cells adhere on the substrate, the nucleoli predominate the coordinates of the maximum phase contrast. As the nucleoli are located in the nucleus, the resulting $x, y$-values can be expected to be a good approximation of the cell center. The influence of cellular organelles other than the nucleoli is expected to be small due to the applied smooth filter of $5 \times 5$ pixels. This is supported by the small fluctuations in the $x, y$-trajectories in Fig. 4 as well as by the fact that in the phase contrast images in Fig. 1, the cellular organelles other than the nucleoli appear only with a marginal contrast. 

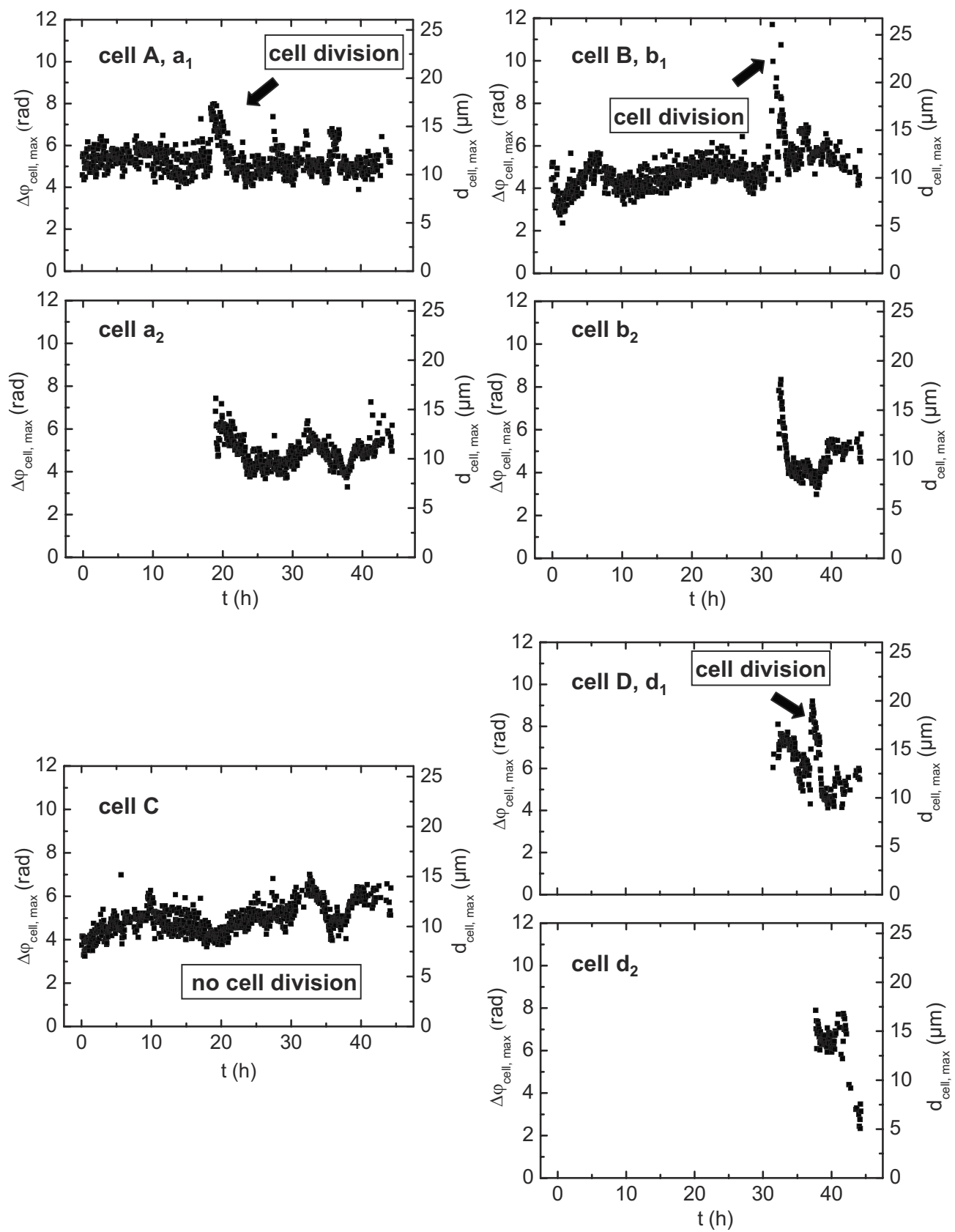

Fig. 3 Time-dependent maximum phase contrast $\Delta \varphi_{\text {cell,max }}$ and time-dependent corresponding maximum cell thickness $d_{\text {cell,max }}$ obtained from the quantitative contrast images in Fig. 1.

During the cell rounding process, the maximum phase contrast is well defined by the center of the resulting spherical structure. Thus, also for this period of the cell cycle, a reliable detection of the cell center is possible. For the phases of the cell cycle in which the cells adhere on the substrate with thin morphology, our algorithm is expected to be more efficient than the evaluation of the DHM phase contrast images for two-dimensional cell tracking by classical edge detection algorithms. During the periods when the cells adhere, the thickness of the outer cell border areas is $\leqslant 1$ to $2 \mu \mathrm{m}$. Consequently, the cells appear with a low contrast of the boundaries in the DHM phase contrast images (see Fig. 1), which would affect the robustness of an edge detection-based determination of the lateral cell position.

Following the approach of Barer, ${ }^{31}$ additional quantitative temporally resolved information about a concentration change of the intracellular substances may be obtained by dry mass measurements, as proposed previously. ${ }^{32,33}$ However, this requires further research on the development and optimization of suitable robust image processing algorithms.

In conclusion, the data in Figs. 1-4 demonstrate the applicability of DHM for quantitative monitoring of the cell division processes during long-term time-lapse observations. DHM provides an efficient method for automated cell thick- 

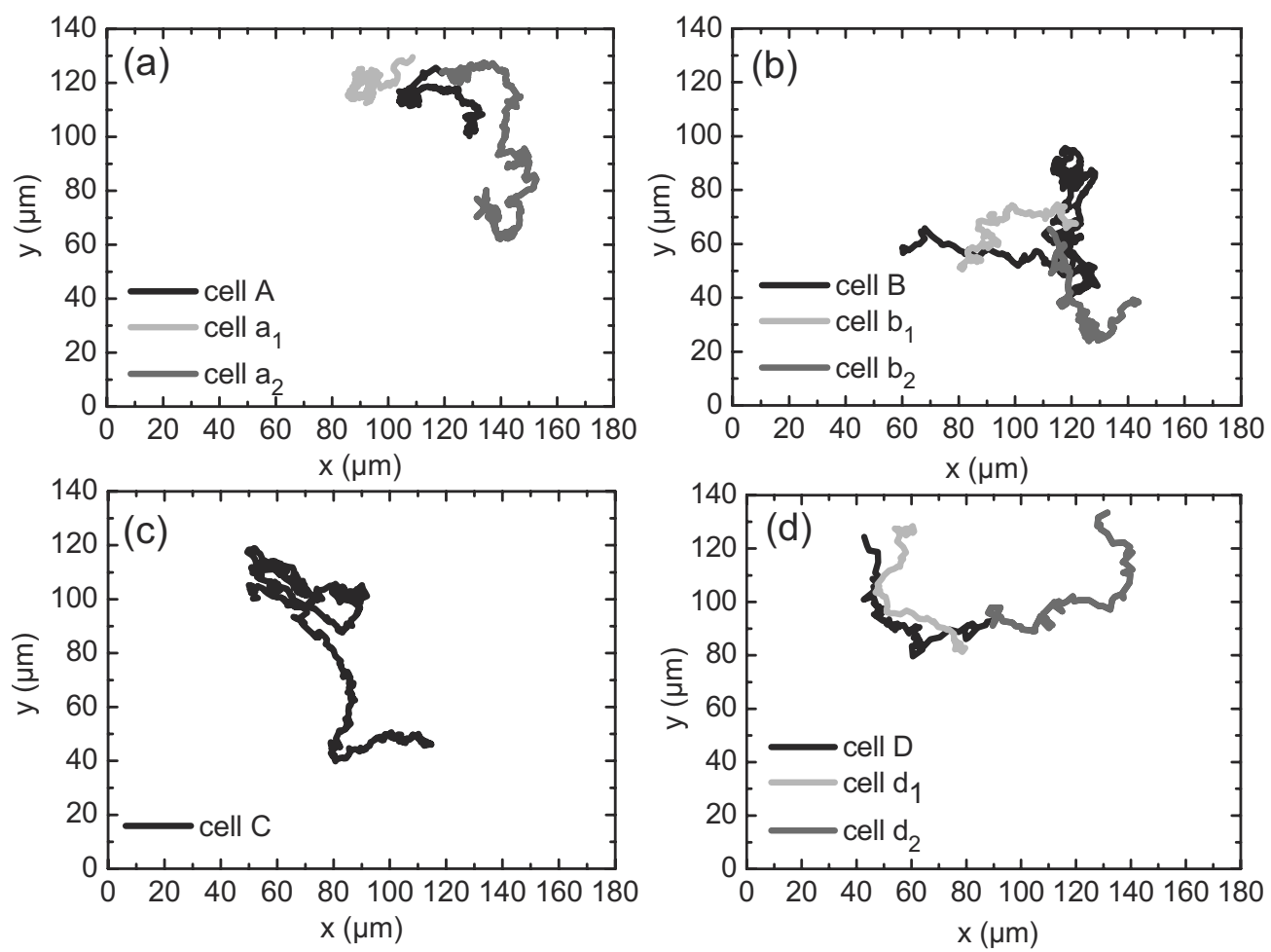

Fig. 4 Two-dimensional quantitative tracking of the cells in the DHM phase-contrast images in Fig. 1. (a) Trajectories of cell A and daughter cells $\mathrm{a}_{1}, \mathrm{a}_{2}$ after cell division; (b) trajectories of cell $\mathrm{B}$ and daughter cells $\mathrm{b}_{1}, \mathrm{~b}_{2}$ after cell division; (c) trajectory of cell C (no cell division is observed); and (d) trajectories of cell $\mathrm{D}$ and daughter cells $\mathrm{d}_{1}, \mathrm{~d}_{2}$ after cell division.

ness monitoring, while simultaneously the two-dimensional cell migration trajectories are obtained. Furthermore, subcelluar regions with a higher molecular density than that of the surrounding cell compartments, such as nucleus and nucleoli, are visible in the phase contrast images. Possible applications of the method include the minimally invasive quantification of cell vitality in the research fields of toxin-mediated cell damage and tumor cell migration analysis in cancer research.

\section{Acknowledgments}

This work was supported by grants from the German Federal Ministry of Education and Research (BMBF), project FKZ 13N9270 within the research focus program Biophotonics; the Deutsche Forschungsgemeinschaft (DFG), program Infections of the Endothelium SPP 1130 project KA 717/4-3; the Graduiertenkolleg GRK 1409; and by grants from the Interdisciplinary Center of Clinical Research (IZKF) Münster, Project No. Me2/023/08.

\section{References}

1. E. Cuche, P. Marquet, and C. Depeursinge, "Simultaneous amplitudecontrast and quantitative phase-contrast microscopy by numerical reconstruction of fresnel off-axis holograms," Appl. Opt. 38, 66947001 (1999)

2. D. Carl, B. Kemper, G. Wernicke, and G. von Bally, "Parameteroptimized digital holographic microscope for high-resolution livingcell analysis," Appl. Opt. 43, 6536-6544 (2004).

3. P. Marquet, B. Rappaz, P. J. Magistretti, E. Cuche, Y. Emery, T. Colomb, and C. Depeursinge, "Digital holographic microscopy: a noninvasive contrast imaging technique allowing quantitative visualization of living cells with subwavelength axial accuracy," Opt. Lett. 30, 468-470 (2005).

4. C. J. Mann, L. Yu, C.-M. Lo, and M. K. Kim, "High-resolution quan- titative phase-contrast microscopy by digital holography," Opt. Express 13, 8693-8698 (2005).

5. F. Charrière, J. Kühn, T. Colomb, F. Montfort, E. Cuche, Y. Emery, K. Weible, P. Marquet, and C. Depeursinge, "Characterization of microlenses by digital holographic microscopy," Appl. Opt. 45, 829835 (2006).

6. B. Kemper and G. von Bally, "Digital holographic microscopy for live cell applications and technical inspection," Appl. Opt. 47, A52A61 (2008).

7. G. von Bally, B. Kemper, D. Carl, S. Knoche, M. Kempe, C. Dietrich, M. Stutz, R. Wolleschensky, K. Schütze, M. Stich, A. Buchstaller, K. Irion, J. Beuthan, I. Gersonde, and J. Schnekenbuger, "New methods for marker-free live cell and tumor analyis," in Biophotonics: Vision for Better Healthcare, J. Popp and M. Strehle, Eds., pp. 301-360, Wiley-VCH, Weinheim (2006).

8. B. Kemper, D. Carl, A. Höink, G. von Bally, I. Bredebusch, and J. Schnekenburger, "Modular digital holographic microscopy system for marker free quantitative phase contrast imaging of living cells," Proc. SPIE 6191, 61910T (2006).

9. L. G. Alexopoulos, G. R. Erickson, and F. Guilak, "A method for quantifying cell size from differential interference contrast images: validation and application to osmotically stressed chondrocytes," $J$. Microsc. 205, 125-135 (2001).

10. A. Barty, K. A. Nugent, D. Paganin, and A. Roberts, "Quantitative optical phase microscopy," Opt. Lett. 23, 817-819 (1998).

11. G. Popescu, L. P. Deflores, J. C. Vaughan, K. Badizadegan, H. Iwai, R. R. Dasari, and M. S. Feld, "Fourier phase microscopy for investigation of biological structures and dynamics," Opt. Lett. 29, 23992401 (2004).

12. T. Ikeda, G. Popescu, R. R. Dasari, and M. S. Feld, "Hilbert phase microscopy for investigating fast dynamics in transparent systems," Opt. Lett. 30, 1165-1167 (2005).

13. J. Farinas and A. S. Verkman, "Cell volume and plasma membrane osmotic water permeability in epithelial cell layers measured by interferometry," Biophys. J. 71, 3511-3522 (1996).

14. P. Tychinskii, "Dynamic phase microscopy: is a dialogue with the cell possible?" Phys. Usp. 50, 513-528 (2007).

15. V. P. Tychinskii, "Coherent phase microscopy of intracellular pro- 
cesses," Phys. Usp. 44, 617-629 (2001).

16. A. D. Aguirre, P. Hsiung, T. H. Ko, I. Hartl, and J. G. Fujimoto, "High-resolution optical coherence microscopy for high-speed, in vivo cellular imaging," Opt. Lett. 28, 2064-2066 (2003).

17. E. A. Swanson, J. A. Izatt, M. R. Hee, D. Huang, C. P. Lin, J. S. Schuman, C. A. Puliafito, and J. G. Fujimoto, "In vivo retinal imaging by optical coherence tomography," Opt. Lett. 18, 1864-1866 (1993).

18. Y. Zhao, Z. Chen, Z. Ding, H. Ren, and J. S. Nelson, "Real-time phase-resolved functional optical coherence tomography by use of optical Hilbert transformation," Opt. Lett. 27, 98-100 (2002).

19. C. G. Rylander, D. P. Davé, T. Akkin, T. E. Milner, K. R. Diller, and A. J. Welch, "Quantitative phase-contrast imaging of cells with phase-sensitive optical coherence microscopy," Opt. Lett. 29, 15091511 (2004).

20. A. K. Ellerbee, T. L. Reazzo, and J. A. Izatt, "Investigating nanoscale cellular dynamics with cross-sectional spectral domain phase microscopy," Opt. Express 15, 8115-8124 (2007).

21. C. Joo, K. H. Kim, and J. F. de Boer, "Spectral-domain optical coherence phase and multiphoton microscopy," Opt. Lett. 32, 623-625 (2007).

22. P. Langehanenberg, B. Kemper, D. Dirksen, and G. von Bally, "Autofocusing in digital holographic phase contrast microscopy on pure phase objects for live cell imaging," Appl. Opt. 47, D176-D182 (2008).

23. P. Langehanenberg, B. Kemper, and G. von Bally, "Autofocus algorithms for digital-holographic microscopy," Proc. SPIE 6633 66330E (2007).

24. P. Langehanenberg, L. Ivanova, I. Bernhardt, S. Ketelhut, A. Vollmer, D. Dirksen, G. Georgiev, G. von Bally, and B. Kemper, "Automated three-dimensional tracking of living cells by digital holographic microscopy," J. Biomed. Opt. 14, 014018 (2009).

25. M. F. Stins, F. Gilles, and K. S. Kim, "Selective expression of adhesion molecules on human brain microvascular endothelial cells," $J$ Neuroimmunol. 76, 81-90 (1997).
26. B. Kemper, D. Carl, J. Schnekenburger, I. Bredebusch, M. Schäfer, W. Domschke, and G. von Bally, "Investigation of living pancreas tumor cells by digital holographic microscopy," J. Biomed. Opt. 11, 034005 (2006).

27. S. Kosmeier, B. Kemper, P. Langehanenberg, I. Bredebusch, J. Schnekenburger, A. Bauwens, and G. von Bally, "Determination of the integral refractive index of cells in suspension by digital holographic phase contrast microscopy," Proc. SPIE 6991, 699110 (2008).

28. B. Kemper, S. Kosmeier, P. Langehanenberg, G. von Bally, I. Bredebusch, W. Domschke, and J. Schnekenburger, "Integral refractive index determination of living suspension cells by multifocus digital holographic phase contrast microscopy," J. Biomed. Opt. 12, 054009 (2007).

29. B. Rappaz, P. Marquet, E. Cuche, Y. Emery, C. Depeursinge, and P. Magistretti, "Measurement of the integral refractive index and dynamic cell morphotometry of living cells with digital holographic microscopy," Opt. Express 13, 9361-9373 (2005).

30. B. Rappaz, F. Charrière, C. Depeursinge, J. Magistretti, and P. Marquet, "Simultaneous cell morphometry and refractive index measurement with dual-wavelength digital holographic microscopy and dyeenhanced dispersion of perfusion medium," Opt. Lett. 33, 744-746 (2008).

31. R. Barer, "Interference microscopy and mass determination," Nature (London) 169, 366-367 (1952).

32. G. Popescu, Y. Park, N. Lue, C. Best-Popescu, L. Deflores, R. R Dasari, M. S. Feld, and K. Badizadegan, "Optical imaging of cell mass and growth dynamics," Am. J. Physiol.: Cell Physiol. 295, C538-544 (2008).

33. B. Rappaz, E. Canob, T. Colomb, J. Kühn, V. Simanis, C. Depeursinge, P. J. Magistretti, and P. Marquet, "Noninvasive characterization of the fission yeast cell cycle by monitoring dry mass with digital holographic microscopy," J. Biomed. Opt. 14, 034049 (2009). 